All TB patients in Arkhangelsk region were tested with molecular-genetic tests before the treatment enabling quicker diagnostics and earlier treatment initiation. Early diagnosis ensures proper treatment regimen for patients with TB and NTM diseases. As a result management of TB patients is improved leading to better treatment outcomes and subsequently reduced TB transmission in the region.

\subsection{5}

doi: 10.15789/2220-7619-2018-4-6.15

\section{DETECTION OF EXTRACELLULAR MYCOBACTERIUM TUBERCULOSIS SMALL RNAS}

M.V. Fursov, T.I. Kombarova, I.A. Dyatlov, V.D. Potapov

State Research Center for Applied Microbiology and Biotechnology, Obolensk, Russia

According to WHO, tuberculosis infection is one of the top ten deadly infections in the world, and about one-fourth of the human population is a carrier of latent tuberculosis infection (LTBI) without manifestation of disease symptoms. The LTBI associated with the conversion of $M$. tuberculosis bacilli to a dormant, metabolically inactive state; however, the molecular mechanisms of this change is not well studied. In many researches the small RNAs (sRNAs) was proposed as regulators of these processes. The aim of the study was detection of sRNA transcripts in cultural supernatant of $M$. tuberculosis strain $\mathrm{H} 37 \mathrm{Rv}$ and into the blood serum of mice $(\mathrm{C} 57 \mathrm{Bl})$ infected with LTBI.

Mycobacterial cells were grown in Middlebrook $7 \mathrm{H} 9$ containing $10 \% \mathrm{ADC}$ supplement at $37^{\circ} \mathrm{C}$ and harvested at different growth phase for use. The culture of M. tuberculosis was centrifuged at $6000 \mathrm{~g}$ for $20 \mathrm{~min}$ at $4^{\circ} \mathrm{C}$. The supernatant was filtered through $0.22 \mu \mathrm{m}$ filters to remove the remaining bacteria. Bacterial total RNA was extracted from M. tuberculosis cultural supernatant by ExtractRNA reagent (Evrogen, Moscow, Russia), followed by digestion with Turbo DNase-free kit (Ambion, Austin, TX, USA) to remove contaminating DNA. cDNA was synthesized with $1.5 \mu \mathrm{g}$ of total RNA by M-MLV (Evrogen, Moscow, Russia) transcriptase and random hexadeoxynucleotides according to the manufacture's instruction. The quantitative RT-PCR (qPCR) was carried out with the qPCRmix-HS SYBR (Evrogen, Moscow, Russia) and the CFX-96 real-time PCR detection system (BioRad, USA). All primers used in this study were designed using VectorNTI 11 (Invirogen, USA) and GeneRunner software (http://www.generunner.net) and synthesized at Evrogen (Moscow, Russia). LTBI C57B1 mouse model was designed as previously described (Shramko et al., 2010). The certain sRNA transcripts have been detected in $M$. tuberculosis culture supernatant at different growth phases (exponential phase, stationary phase and late-stationary phase) and into the blood serum of mice infected with LTBI. Obtained data allow us to propose M. tuberculosis sRNAs as new markers for LTBI diagnostic in the future.

\section{TB PORTALS PROGRAM: DATA-DRIVEN MULTI- NATIONAL CONSORTIUM AGAINST DRUG-RESISTANT TUBERCULOSIS}

\section{A.E. Gabrielian, A. Rosenthal}

Office of Cyber Infrastructure and Computational Biology, National Institute of Allergy and Infectious Diseases, National Institutes of Health, Rockvill, MD, USA

TB Portals Program (TBPP) is an international initiative that is founded, developed and steered by doctors, researchers, IT specialists and radiologists to combat drug-resistant tuberculosis (DR-TB). The efforts of hospitals and biomedical research institutes from ten countries on three continents are organized and supported by TBPP to actively establish and grow an integrated open-access network of innovative tools and data from real patient cases of TB (tbportals.nid.nih.gov). The TB Portals collect, analyze, standardize, and present anonymized clinical, laboratory, and socioeconomic data, full bacterial genomes, and radiological data (CXR and CT).

The TBPP database currently has more than 1300 published (22 250 total cases), $75 \%$ of which are MDR or XDR-TB. Clinicians supply and validate all patient data. Once validated, the data become published with open access status in accordance with NIH FAIR principles. Importantly, both original and derived (expert-based and computational annotations) data remain patient-centric, i.e. linked to a unique patient identifier. This cornerstone principle enables users to define and analyze cohorts of patients, augmenting OMICS studies with diverse clinical information.

To date the database contains 730 published (1300 total) fully sequenced and annotated Mycobacterium tuberculosis genomes associated with the patient case record. We will highlight several TBPP projects studying 1) genomic signatures for TB relapse and reinfection, and 2) comparative analysis of M. tuberculosis strains isolated from sputum vs. surgically removed parts of lungs.

TBPP assists doctors and researchers in testing and refining their hypotheses with friendly and powerful tools. Starting from genomics and the molecular basis of drug resistance, we will demonstrate how our online tools enable anyone to simultaneously look at clinical, microbiological and radiological evidences in order to (1) search for genomic clues for inconsistencies in existing DR-TB diagnostics and to (2) study common and countries-specific evolutionary patterns of $M$. tuberculosis.

6.17 doi: 10.15789/2220-7619-2018-4-6.17

\section{SIMPLIFYING NGS APPROACHES TO OPTIMIZE TRACING OF TRANSBORDER SPREAD OF MYCOBACTERIUM TUBERCULOSIS}

\section{Garcia de Viedma}

Clinical Microbiology and Infectious diseases Department, Gregorio Marañón University Hospital, Madrid, Spain; CIBER Enfermedades Respiratorias CIBERES, Madrid, Spain

Molecular epidemiology, and more recently genomic epidemiology, based on whole genome sequencing (WGS), improve our understanding about the transmission dynamics of Mycobacterium tuberculosis in a population. However, in many countries, including many of those with a high burden of TB, systematic genomic epidemiology cannot be implemented. Trying to find a solution to this situation, we propose an alternative line of progression, which tries to conciliate the discriminatory power of WGS with the speed, low cost and simplicity of PCR-based approaches. The cost of this shortcut is that it sacrifices the complete knowledge of all the transmission clusters in a population, because it needs to focus on surveying the strains that deserve special attention because they are more actively transmitted, or correspond to high-risk MDR or XDR strains. This short-cut strategy has proved to be efficient to survey actively transmitted strains, to fast track outbreak-strains, to update the presence of high-risk strains in a population or to give an urgent answer to public-health alerts, such as to rule out secondary cases due to the importation of XDR-TB cases. More recently, we are integrating this strategy to optimize the characteriza- 